\title{
Neurological Critical Care Services' Influence Following Large Hemispheric Infarction and Their Impact on Resource Utilization
}

\author{
Syed Omar Shah1,2,3,4*, Yu Kan Au¹, Fred Rincon1,2,3,4, Matthew Vibbert1,2,3,4 \\ 1 Department of Neurology, Thomas Jefferson University, Philadelphia, PA USA \\ 2 Department of Neurosurgery, Thomas Jefferson University, Philadelphia, PA USA \\ ${ }^{3}$ Divisions of Critical Care and Neurotrauma, Thomas Jefferson University, Philadelphia, PA USA \\ ${ }^{4}$ Department of Cerebrovascular Diseases, Thomas Jefferson University, Philadelphia, PA USA
}

\begin{abstract}
Introduction: Acute ischemic stroke (AIS) is the fourth leading cause of death in the US. Numerous studies have demonstrated the use of comprehensive stroke units and neurological intensive care units (NICU) in improving outcomes after stroke. We hypothesized that an expanded neurocritical care (NCC) service would decrease resource utilization in patients with LHI.

Methods: Retrospective data from consecutive admissions of large hemispheric infarction (LHI) patients requiring mechanical ventilation were acquired from the hospital medical records. Between 2011-2013, there were 187 consecutive patients admitted to the Jefferson Hospital for Neuroscience (Philadelphia, USA) with AIS and acute respiratory failure. Our intention was to determine the number of tracheostomies done over time. The primary outcome measure was the number of tracheostomies over time. Secondary outcomes were, ventilator-free days (Vfd), total hospital charges, intensive care unit length of stay (ICU-LOS), and total hospital length of stay (hospital-LOS), including ICU LOS. Hospital charges were log-transformed to meet assumptions of normality and homoscedasticity of residual variance terms. Generalized Linear Models were used and ORs and $95 \% \mathrm{Cls}$ calculated. The significance level was set at $\alpha=0.05$.
\end{abstract}

Results: Of the 73 patients included in this analysis, 33\% required a tracheostomy. There was a decrease in the number of tracheostomies undertaken since 2011. (OR 0.8; 95\% Cl 0.6-0.9: $\mathrm{p}=0.02$ ).

Lower Vfd were seen in tracheostomized patients (OR 0.11; 95\% Cl 0.1-0.26: $\mathrm{p}<0.0001$ ). The log-hospital charges decreased over time but not significantly (OR $0.9 ; 95 \% \mathrm{Cl} 0.78-1.07: \mathrm{p}=0.2)$ and $(\mathrm{OR} 0.99 ; 95 \% \mathrm{Cl} 0.85-1.16: \mathrm{p}=0.8)$ from 2012 to 2013 respectively.

The ICU-LOS at 23 days vs 10 days ( $p=0.01$ ) and hospital-LOS at 33 days vs 11 days $(p=0.008)$ were higher in tracheostomized patients.

Conclusion: The data suggest that in LHI-patients requiring mechanical ventilation, a dedicated NCC service reduces the overall need for tracheostomy, increases Vfd, and decreases ICU and hospital-LOS.

Keywors: neurocritical care, neurological critical care, large hemispheric infarct, malignant stroke, extubation, tracheostomy, resource utilization

Received: 18 June 2017 / Accepted: 12 December 2017

Correspondence to: Syed Omar Shah, Department of Neurological Surgery, Thomas Jefferson University and Jefferson College of Medicine. 909 Walnut Street, 3rd Floor, Philadelphia, PA 19107. E-Mail:syed.o.shah@jefferson.edu 


\section{INTRODUCTION}

Not specific to the neurologically injured population, it is estimated that between 5 and $20 \%$ of patients undergoing extubation will experience extubation failure, defined as re-intubation within 48 hours of extubation [1]. Current guidelines developed by the American College of Chest Physicians (ACCP), the American Association for Respiratory Care (AARC), and the American College of Critical Medicine (ACCM) recommend extubation if the following are met [2]:

- evidence for some reversal of the underlying cause for respiratory failure

- adequate oxygenation with a $\mathrm{PaO} 2 / \mathrm{Fio} 2$ ratio $>150$ to 200

- the patient requiring positive end-expiratory pressure $[\mathrm{PEEP}] \leq 5$ to $8 \mathrm{~cm} \mathrm{H} 2 \mathrm{O}$

- Fio $2 \leq 0.4$ to 0.5

- $\mathrm{pH} \geq 7.25$

- hemodynamic stability

- the capability of initiating an inspiratory effort.

Unfortunately, these guidelines can be difficult to follow for patients who are intubated for neurological reasons rather than primary lung pathology. Additionally, traditional respiratory weaning parameters may not predict extubation in neurological critical care patients [3].

Patients with large hemispheric infarction (LHI) represent a subset of critically ill, neurologically injured patients that present challenges for extubation. There are no large studies that are applicable in terms of extubation or weaning protocols. Previous studies in neurological patients reported mixed results as to whether low GCS scores predict extubation failure; the FOUR score failed to predict extubation failure in one study [3-7]. The Neurocritical Care Society published guidelines recommending that extubation should be attempted in patients who demonstrate successful spontaneous breathing trials, an absence of excessive oropharyngeal saliva, an absence of need of frequent suctioning, a presence of a cough reflex and tube intolerance. Additionally, patients should not be receiving analgesic or sedation medication. [4]. These recommendations however are based on very low quality of evidence.

Evidence suggests that outcomes are better when critically ill neurological patients are treated by a dedicated NCC team. [8-10]. Beginning in September,
2010, Jefferson Hospital for Neuroscience (Philadelphia, USA) effected a number of organizational changes aimed at improving care for patients admitted with AIS:

- AIS patients with a large hemispheric infarction (LHI) were admitted to a dedicated neuroscience hospital.

- NICU service coverage was expanded to include ICU coverage by neurointensivist with no other clinical duties outside of the NICU seven days a week. This increase in neurointensivist resources reduced the average number of ICU beds covered by a single attending physician from 26 to 13 .

- daily assessments for discontinuation of mechanical ventilation were routinely included in daily multidisciplinary rounds including protocolized performance of daily spontaneous awakening trials and spontaneous breathing trials $[12,13]$.

These changes were full implemented by January 2011. It was hypothesized that an expanded NICU service would decrease resource utilization including the number of tracheostomies placed and the length of stay of LHI patients within the ICU. The purpose of this study was to determine if these organizational changes would have an impact on our resource utilization.

\section{METHODS}

A retrospective cohort analysis was conducted with the written approval of the Institutional Review Board of Thomas Jefferson University Hospital (IRB \#14D.66). Admissions with a primary diagnosis of acute ischemic stroke or with acute respiratory failure (ARF) were identified from the hospital discharge database. From January 2011 to December 2013, 187 consecutive patients with AIS also had ARF. Patients that had computed tomography (CT) ischemic changes affecting more than two-thirds of the MCA territory and including the basal ganglia along with space-occupying edema, were included. Patients were excluded who met following criteria: age less than 18 years, performance of tracheostomy without a preceding attempted extubation, withdrawal of life sustaining therapies due to institution of comfort care, and diagnosis of brain death. After application of the inclusion and exclusion criteria, 73 patients were included in this analysis.

Patients' age, race, BMI, gender, past medical history, NIHSS, and GCS were abstracted from hospital records. APACHE2 scores were calculated from ad- 
mission values abstracted from the hospital record. Head CTs were reviewed by investigators to confirm infarct size and assign ASPECTS scores. The dates of intubation and extubation, tracheostomy, ICU length of stay, hospital length of stay, ventilator free days and total hospital charges was also collected. The primary outcome measure was the number of tracheostomies over time. Secondary outcomes were, ventilator-free days (Vfd), total hospital charges, and the ICU-LOS and hospital LOS.

\section{Statistical Analysis}

Bivariate comparisons were made using the $t$-test or Mann-U-Whitney test for continuous variables and $\chi^{2}$ or Fisher's exact test for categorical variables. Hospital charges were log-transformed to meet assumptions of normality and homoscedasticity of residual variance terms. ORs and 95\% CIs were calculated using Generalized Linear Models. The significance level was set at $\alpha=0.05$.

Statistical analyses were undertaken using JMP software version 11.0 (SAS, Cary NC).

\section{RESULTS}

Demographics, admission severity scores and stroke presentation are summarized in Table 1. Patients who required tracheostomy were older. The mean (Standard deviation [SD]) age were 61(14) for patients who did not require tracheostomy and 68(8) for patients who required tracheostomy (61, SD (14) vs. $68, \mathrm{SD}$ (9), $\mathrm{p}=0.01$ ). Patients who required tracheostomy had higher admission APACHE2 scores than those patients who did not require tracheostomy. The median (interquartile range [IQR]) APACHE2 scores were 16(1319) for patients who did not require tracheostomy and 21(16-24) for patients who required tracheostomy (16, interquartile range [IQR] (13-19) vs. 21, IQR (16-24),

Table 1. Demographic, stroke symptoms and severity indexes for tracheostomy and no tracheostomy group

\begin{tabular}{|c|c|c|c|c|}
\hline & $\begin{array}{c}\text { All } \\
\mathrm{N}=73\end{array}$ & $\begin{array}{l}\text { No Tracheostomy } \\
\qquad N=49\end{array}$ & $\begin{array}{l}\text { Tracheostomy } \\
\qquad N=24\end{array}$ & P Value \\
\hline \multicolumn{5}{|l|}{ Epidemiology } \\
\hline Age, $X(S D)$ & $63(12)$ & $61(14)$ & $68(9)$ & $* 0.01$ \\
\hline Race, White, N (SD) & $41(50)$ & $29(70)$ & $12(29)$ & 0.44 \\
\hline BMI & $29(12)$ & $29(6)$ & $29(9)$ & 0.7 \\
\hline Gender, Female & $28(41)$ & $15(50)$ & $13(50)$ & 0.9 \\
\hline \multicolumn{5}{|l|}{ PMHx } \\
\hline AIS & $12(51)$ & $5(42)$ & $7(58)$ & 0.06 \\
\hline Hypertension & $55(45)$ & $34(62)$ & $21(38)$ & 0.07 \\
\hline Dyslipidemia & $29(40)$ & $21(72)$ & $8(27)$ & 0.6 \\
\hline Diabetes Mellitus & $30(51)$ & $19(63)$ & $11(37)$ & 0.33 \\
\hline Atrial Fibrillation & $13(48)$ & $7(53)$ & $6(46)$ & 0.26 \\
\hline COPD & $11(51)$ & $6(55)$ & $5(45)$ & 0.48 \\
\hline $\mathrm{CHF}$ & $14(42)$ & $7(50)$ & $7(50)$ & 0.12 \\
\hline \multicolumn{5}{|l|}{ Stroke } \\
\hline Presence of Aphasia, N (\%) & $38(52)$ & $25(51)$ & $13(54)$ & 0.8 \\
\hline Presence of Neglect, N (\%) & $37(50)$ & $27(55)$ & $10(41)$ & 0.3 \\
\hline Decompressive Hemicraniectomy, N (\%) & $31(42)$ & $23(47)$ & $8(33)$ & 0.3 \\
\hline \multicolumn{5}{|l|}{ Severity Scores on Admission } \\
\hline APACHE 2, med (IQR) & $17(13-21)$ & $16(13-19)$ & $21(16-24)$ & *0.002 \\
\hline ASPECTS, med (IQR) & $4(1-6)$ & $3(0-6)$ & $5(2-6)$ & 0.28 \\
\hline NIHSS, med (IQR) & $17(14-22)$ & $17(14-22)$ & $19(15-22)$ & 0.53 \\
\hline GCS, med (IQR) & $10(9-13)$ & $10(9-14)$ & $10(9-13)$ & 0.87 \\
\hline
\end{tabular}


$\mathrm{p}=0.002$ ). Admission GCS, NIHSS, and ASPECTS scores were similar between the two groups.

The results were summarized in Table 2. Of the 73 study patients, 24 patients (33\%) required a tracheostomy. Overall, the number of tracheostomies decreased over time from 2011 to 2013 ( $\mathrm{p}=0.02$ ). The trend of tracheostomies is summarized in Figure 1. A 21\% reduction in tracheostomies per year was observed since 2011.

Fewer ventilator free days were seen in tracheostomized patients $(\mathrm{p}<0.0001)$. The mean hospital costs of $\$ 452,000$ for the tracheostomy group was significantly higher compared to the costs of $\$ 299,000$ for extubated patients $(\mathrm{p}=0.02)$ (Figure 2). Adjusted loghospital costs decreased over time from 2011 to 2013 but not significantly.

Finally, both the ICU stay (23 days vs. 10 days, $\mathrm{p}=0.01$ ) and hospital-LOS (33 days vs. 11 days, $\mathrm{p}=0.008)$ were higher in tracheostomized patients as compared to extubated patients (Figure 3 ) during the study period from 2011 to 2013.

\section{DISCUSSION}

The principal findings suggest that in LHI patients requiring mechanical ventilation, a dedicated NCC service improves the rates of weaning off from ventilator by reducing the overall need for tracheostomy, increases Vfd, and decreases ICU and hospital-LOS.

The American Heart Association guidelines (2014) concerning management of AIS patients with brain swelling recommends that patients with LHI should be admitted to a dedicated neurologic intensive care or stroke unit with care provided by an attending physician with training in neurologic critical care or vascular neurology [15]. We infer from the recommendations that all ventilated patients with LHI should be admitted to a neurologic intensive care unit. Studies have demonstrated a benefit in mortality, length of stay, and discharge disposition on neurological critically ill patients that were treated in a NICU. $[8,10,16]$.

Other studies have demonstrated a benefit for a NCC service in the setting of AIS. To our knowledge, none

Table 2. Primary outcome measures for tracheostomy and no tracheostomy group

\begin{tabular}{lccc} 
& All (N=73) & No Tracheostomy (N=49) & Tracheostomy (N=24) \\
Extubation vs Tracheostomy Over Time & & & \\
$2011, N$ & 33 & 18 & 15 \\
$2012, N$ & 19 & 12 & 7 \\
$2013, N$ & 21 & 19 & 2 \\
\hline
\end{tabular}

\section{Ventilator Days}

2011-2013, X (SD)*

$9.7(3.6)$

$6.3(6.8)$

$14.6(19.6)$

Hospital Costs (USD)
$2011-2013, X(S D, K)^{*}$
$2011, X(S D, K)$
$2012, X(S D, K)$
$2013, X(S D, K)$

$349,292(244)$

419,141 (342)

312,567 (138)

282,907 (78)

\section{ICU Length of Stay (days)}

2011-2013, X (SD)*

2011, X (SD)

2012, X (SD)

2013, X (SD)

\section{Hospital Length of Stay (days)}

$\begin{array}{lr}2011-2013, \times(S D)^{*} & 23(17.5) \\ 2011, X(S D) & 18(9.9) \\ 2012, X(S D) & 22(16.7) \\ 2013, X(S D) & 27(21.2\end{array}$

$14(14.5)$

$11(8.7)$

$12(7.7)$

18 (19.7)
$\$ 452,217$ (358)

$505,792(94.7)$

362,475 (139)

364,503 (259)

$\begin{array}{ll}\$ 298,880(143) & \$ 452,217(358) \\ 338,223(33.6) & 505,792(94.7) \\ 269,730(41.1) & 362,475(139) \\ 280,019(32.7) & 364,503(259)\end{array}$

SD - Standard Deviation, K- in thousands of USD, USD - US Dollars, $\mathrm{N}$ - Number, $\mathrm{X}$ - Average, * significant at $\alpha$ level 0.05 . 




Fig. 1. Extubations Versus Tracheostomy in LHI Patients with ARF Over Time.

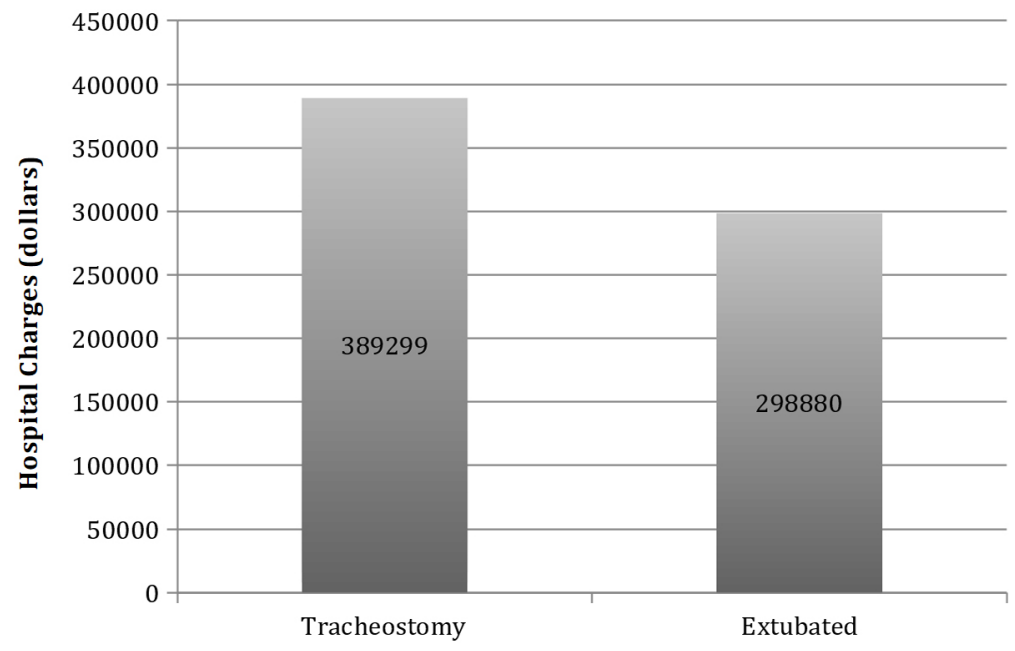

Fig. 2. Mean Total Hospital Costs of Extubated and Tracheostomy patients.

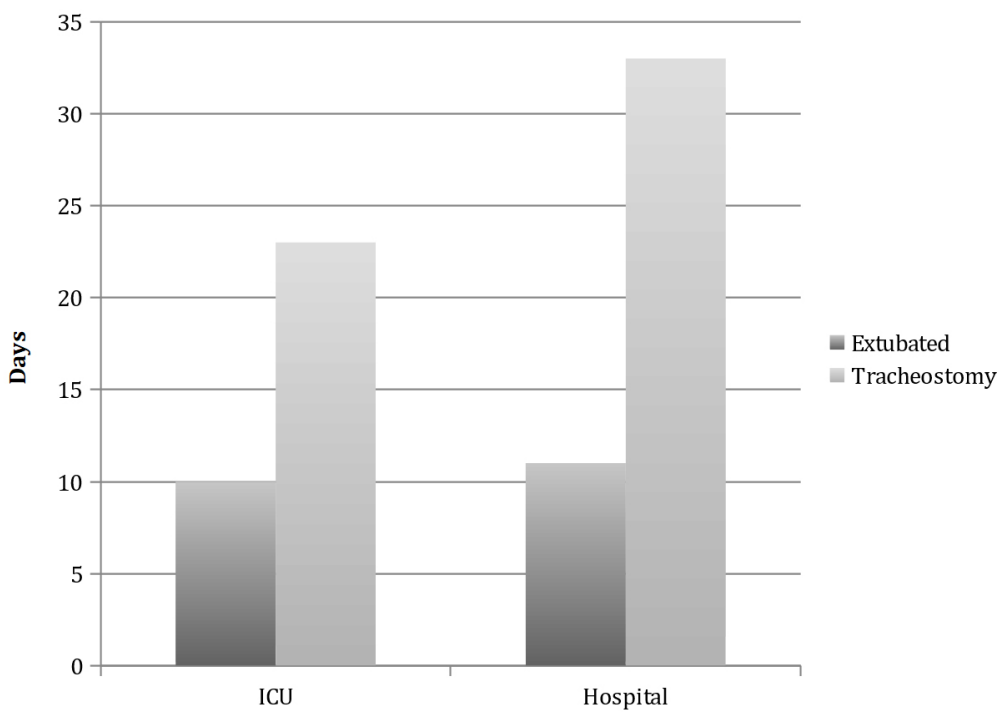

Fig. 3. Total ICU and Hospital Stay Days for Extubated and Tracheostomy Patients 
have described the impact of a NCC service on the rate of tracheostomies performance and whether there is an associated benefit with regard to the length of stay in both the ICU-LOS or hospital-LOS. Knopf [2012] studied 2,096 AIS patients and found that those treated in an NICU or by a neurologic critical care specialist had lower in-hospital mortality, better discharge disposition, and shorter hospital LOS compared to controls. [17]. Similarly, Varelas [2008] performed a prospective analysis of 433 AIS patients, 259 of which were admitted after a NCC service had been put in place. [9]. Patients in this study who were cared for by an NCC service had a shortened NICU course and hospital stay as well as an improved discharge disposition. Bershad [2008] also reported a reduction in resource utilization and improved patient outcomes for patients with AIS who were treated in a dedicated unit. [18].

Prior investigations have assessed predictors of successful extubation in a neurological ICU, but these studies have all included neurosurgical patients with tumors, subarachnoid hemorrhages, subdural and epidural hemorrhages along with patients with AIS. Moreover, some of these studies also include patients with non-neurological comorbidities. [5-7]. Retrospective data suggest that GCS and the ability to follow commands help predict extubation, but this data was not obtained exclusively from AIS patients. Preliminary data from a pilot study of early tracheostomy in patients with hemorrhagic or ischemic stroke suggests that such patients may have improved survival and long-term functional outcomes, but this is currently being validated with a multicenter clinical trial $[19,20]$.

We believe that the expansion of a NCC service drove the observed reduction in tracheostomies and consequently led to increases in ventilator free days while decreasing ICU and hospital length of stay. Expansion of the NCC service permitted a more time-intensive daily evaluation of readiness for ventilator liberation that included daily formal awakening and spontaneous breathing trials with multidisciplinary input from nursing, pharmacy, and respiratory therapy.

Tracheostomies are unfortunately a frequently necessity in patients with LHI. While criteria for early tracheostomy exist, they have not been studied specifically in LHI patients. Therefore, the decision as to whether or when to carry out a tracheostomy is currently one of individual clinician preference. Two retrospective studies of mixed cerebrovascular patients receiving early tracheostomy suggested better outcome measures as well as reductions in ICU stay and costs. [19-21]. There is insufficient evidence for any predictors of tracheostomy for LHI, but some have been associated for patients with intracerebral hemorrhage. [22] We believe that in this patient population, a dedicated neurocritical care specialist is best equipped to treat patients with severe strokes and to evaluate and decide the patient's need for extubation.

There are limitations to the present study. First, this is a retrospective analysis, and thus can only demonstrate an association between the reduction in tracheostomy in patients serviced by a dedicated NCC team and not a causal relationship. Second, no analysis of any short term or long term mortality or functional outcome was undertaken. Thus, we cannot make an assumption that AIS patients who are ventilated will have an overall improvement in functional outcome when treated by a dedicated NCC team. Third, retrospective analysis may be subject to bias inherent to these types of studies. Lastly, there was no set protocol guiding the decision to pursue tracheostomy. Treating physicians made ad hoc decisions regarding the number of attempted extubations and timing of tracheostomy based on clinical factors

This study suggests that LHI patients requiring mechanical ventilation may have an overall decrease in tracheostomy when evaluated by a dedicated NCC service.

A future study of long term follow up of these patients, including a mortality comparison is currently under way.

\section{CONCLUSION}

The data suggest that in LHI patients requiring mechanical ventilation, a dedicated NCC service improves the rates of successful extubation from ventilator rates and reduces the overall need for tracheostomy, increases $\mathrm{V} f d$, and decreases ICU and hospital-LOS.

\section{CONFLICT OF INTEREST}

None to declare

\section{DEFERENCES}

1. Epstein SK. Predicting extubation failure: is it in (on) the cards?. Chest. 2001;120:1061-3.

2. Maclntyre NR. Evidence-based guidelines for weaning and 
Available online at: www.jccm.ro

discontinuing ventilatory support: a collective task force facilitated by the American College of Chest Physicians; the American Association for Respiratory Care; and the American College of Critical Care Medicine. Chest. 2001;120:375S-96S.

3. Ko R, Ramos L, Chalela JA. Conventional weaning parameters do not predict extubation failure in neurocritical care patients. Neurocrit Care. 2009;10:269-73.

4. Torbey MT, Bösel J, Rhoney DH, et al. Evidence-based guidelines for the management of large hemispheric infarction. Neurocrit Care. 2015;22:146-64.

5. Koh WY, Lew TW, Chin NM, Wong MF. Tracheostomy in a neurointensive care setting: indications and timing. Anaesth Intensive Care. 1997;25:365-8.

6. Coplin WM, Pierson DJ, Cooley KD, Newell DW, Rubenfeld GD. Implications of extubation delay in brain-injured patients meeting standard weaning criteria. Am J Respir Crit Care Med. 2000;161:1530-6.

7. Anderson CD, Bartscher JF, Scripko PD, et al. Neurologic examination and extubation outcome in the neurocritical care unit. Neurocrit Care. 2011;15:490-7.

8. Varelas PN, Eastwood D, Yun HJ, et al. Impact of a neurointensivist on outcomes in patients with head trauma treated in a neurosciences intensive care unit. J Neurosurg. 2006;104:713-9.

9. Varelas PN, Schultz L, Conti M, Spanaki M, Genarrelli T, HaceinBey $L$. The impact of a neuro-intensivist on patients with stroke admitted to a neurosciences intensive care unit. Neurocrit Care. 2008;9:293-9.

10. Samuels O, Webb A, Culler S, Martin K, Barrow D. Impact of a dedicated neurocritical care team in treating patients with aneurysmal subarachnoid hemorrhage. Neurocrit Care. 2011;14:334-40.

11. Vahedi K, Hofmeijer J, Juettler E, et al. Early decompressive surgery in malignant infarction of the middle cerebral artery: a pooled analysis of three randomised controlled trials. Lancet Neurol. 2007;6:215-22.

12. Kress JP, Pohlman AS, O'Connor MF, Hall JB. Daily interruption of sedative infusions in critically ill patients undergoing mechanical ventilation. N Engl J Med. 2000;342:1471-7.
The Journal of Critical Care Medicine 2018;4(1) • 11

13. Girard TD, Kress JP, Fuchs BD, et al. Efficacy and safety of a paired sedation and ventilator weaning protocol for mechanically ventilated patients in intensive care (Awakening and Breathing Controlled trial): a randomised controlled trial. Lancet. 2008;371:126-34.

14. Von Elm E, Altman DG, Egger M, Pocock SJ, Gøtzsche PC, Vandenbroucke JP, Strobe Initiative. The Strengthening the Reporting of Observational Studies in Epidemiology (STROBE) Statement: guidelines for reporting observational studies. Int J Surg. 2014;12:1495-9.

15. Wijdicks EF, Sheth KN, Carter BS, et al. Recommendations for the management of cerebral and cerebellar infarction with swelling. Stroke. 2014;45:1222-38.

16. Diringer MN, Edwards DF. Admission to a neurologic/ neurosurgical intensive care unit is associated with reduced mortality rate after intracerebral hemorrhage. Crit Care Med. 2001;29:635-40

17. Knopf L, Staff I, Gomes J, McCullough L. Impact of a neurointensivist on outcomes in critically ill stroke patients. Neurocrit Care. 2012;16:63-71.

18. Bershad EM, Feen ES, Hernandez OH, Suri MF, Suarez JI. Impact of a specialized neurointensive care team on outcomes of critically ill acute ischemic stroke patients. Neurocrit Care. 2008;9:287-92.

19. Bösel J, Schiller P, Hook Y, et al. Stroke-Related Early Tracheostomy Versus Prolonged Orotracheal Intubation in Neurocritical Care Trial (SETPOINT). Stroke. 2013;44:21-8.

20. Schönenberger $S$, Niesen WD, Fuhrer $H$, et al, SETPOINT2study group and the IGNITE-study group. Early tracheostomy in ventilated stroke patients: Study protocol of the international multicentre randomized trial SETPOINT2 (Stroke-related Early Tracheostomy vs. Prolonged Orotracheal Intubation in Neurocritical care Trial 2). Int J Stroke. 2016;11:368-79.

21. Rabinstein AA, Wijdicks EF. Outcome of survivors of acute stroke who require prolonged ventilatory assistance and tracheostomy. Cerebrovasc Dis. 2004;18:325-31.

22. Huttner HB, Kohrmann M, Berger C, Georgiadis D, Schwab $\mathrm{S}$. Predictive factors for tracheostomy in neurocritical care patients with spontaneous supratentorial hemorrhage. Cerebrovasc Dis. 2006;21:159-65. 